\title{
Somewhat More than Governors Need to Know about Trigonometry ${ }^{1}$
}

\author{
SKIP GARIBALDI \\ Department of Mathematics \& Computer Science \\ Emory University \\ Atlanta, GA 30322 \\ skip@member.ams.org
}

In school, I had to memorize the values of sine and cosine at the angles $0,30,45,60$, and 90 degrees. This always made me wonder: Why those angles and not others? After all, there is some angle whose sine is, say, 3/4. Why not include it in the table? More generally: What "nice" angles have "nice" values for sine and cosine?

The main goal of this paper is to find answers to these questions "organically" by making naive guesses at the answers and seeing if these guesses are correct. By successively refining our naive guesses, we end up with essentially complete answers. The second goal of this paper is to highlight the connections between trigonometry and Galois theory by using results from a standard course on Galois theory to answer these questions. These things are well known to experts; we aim to popularize them.

\section{The most a governor needs to know}

The third goal of this article is to help out former Governor Jeb Bush of Florida. At a July 2004 appearance to promote state-wide annual testing of students in public schools, a high school student asked him: "What are the angles in a 3-4-5 triangle?" The governor responded "I don't know. 125, 90 ... and whatever remains on 180." [14] Aside from not noticing that 125 and 90 add up to more than 180, it's not such a bad answer. In his favor, the governor remembered that the interior angles add up to 180, something I'm not confident my governor would remember. Also, he got one of the three angles right. Presumably he remembered that there is a right triangle that has sides 3-4-5 and applied the side-side-side congruence theorem to conclude that every 3-4-5 triangle is a right triangle.

Unfortunately, the story gets worse. The high school student then replied: "It's 30$60-90$," which we all know is wrong, because the cosine of 30 degrees is $\sqrt{3} / 2$, not $4 / 5$.

And the story gets yet worse. The AP reporter asserted that the correct answer was 90, 53.1, and 36.9 degrees, which-although not really wrong-does not address the question of whether or not the governor should have known these angles. A later story [16] did better. It quoted a retired math professor who said "I don't think those are very well known angles" and "I wouldn't expect many mathematicians to know that."

A good answer to the student's question is provided by the following theorem:

THE GOVERNOR's THEOREM. If a right triangle has integer side lengths, then the acute angles are irrational, when measured in degrees.

Looking at the trig table we all memorized, we notice that all the angles are integers when measured in degrees, so no one-much less a governor-should be expected to answer the student's question.

\footnotetext{
${ }^{1}$ This is the text for the address at the 2006 MAA State Dinner for Georgia, held at Mercer University in April.
} 
How to prove the theorem? One way is to get it as a corollary of Lehmer's Theorem, which we will prove in the next section.

Remarks. The Governor's Theorem appears as problem 239b in [15]. The theorem is also true if the word "degrees" is replaced by "radians". That is a standard consequence of the Lindemann-Weierstrass Theorem, and is Exercise 1 in $\S 4.13$ of [8].

\section{Degrees}

Since all the angles in the standard trig table are rational when measured in degrees, below we only consider angles of that type.

Naively, we might want to fill our trig tables with angles $\theta$ such that $\theta$ (measured in degrees), $\sin \theta$, and $\cos \theta$ are all rational. But the Governor's Theorem-still to be proved - tells us that there are no such $\theta$ 's with $0<\theta<90^{\circ}$. So we should include more angles in our trig table.

For example, our standard trig table includes familiar friends like 45 degrees, for which sine and cosine are both $1 / \sqrt{2}$, which is not rational. Rather, $1 / \sqrt{2}$ is algebraic, i.e., there is a nonzero polynomial $f(x)$ with rational coefficients such that $f(1 / \sqrt{2})=$ 0 , namely $f(x)=2 x^{2}-1$.

We might ask: What rational angles are such that $\sin \theta$ and $\cos \theta$ are algebraic? The hope would be that these angles $\theta$ would make up a nice trig table. But this doesn't work, because $\sin \theta$ and $\cos \theta$ are always algebraic when $\theta$ is rational. Gauss said that this was well known in Article 337 of his Disquisitiones [4], and indeed we can see it by using multiple-angle formulas (or reading the rest of this section).

So we need a finer notion of "nice" to pick out angles $\theta$ that one would want in a trig table. Recall that the degree of an algebraic number is defined to be the minimum of $\operatorname{deg} f(x)$ as $f(x)$ varies over the nonzero rational polynomials such that $f(r)$ is zero. For example, rational numbers have degree 1 whereas $1 / \sqrt{2}$ and $\sqrt{3} / 2$ have degree 2 .

Write $\theta$ as $360 k / n$ where $k$ and $n$ are relatively prime natural numbers. The famous number-theorist D.H. Lehmer proved in [10]:

LEHMER'S THEOREM. If $n \geq 3$, then the degree of $\cos (360 k / n)$ is $\phi(n) / 2$. If $n$ is positive and $\neq 4$, then the degree of $\sin (360 \mathrm{k} / \mathrm{n})$ is

$$
\begin{cases}\phi(n) & \text { if } \operatorname{gcd}(n, 8)=1 \text { or } 2 \\ \phi(n) / 4 & \text { if } \operatorname{gcd}(n, 8)=4 \\ \phi(n) / 2 & \text { if } \operatorname{gcd}(n, 8)=8 .\end{cases}
$$

In the theorem, the symbol $\phi$ denotes Euler's $\phi$-function. Recall that $\phi(1)$ is defined to be 1 and that $\phi(m n)=\phi(m) \phi(n)$ for relatively prime numbers $m$ and $n$. Finally, for $p$ a prime we have:

$$
\phi\left(p^{e}\right)=p^{e-1}(p-1) .
$$

The first few values of $\phi$ are given by the table

\begin{tabular}{c|llllllll}
$n$ & 1 & 2 & 3 & 4 & 5 & 6 & 7 & 8 \\
\hline$\phi(n)$ & 1 & 1 & 2 & 2 & 4 & 2 & 6 & 4
\end{tabular}

Let us check Lehmer's Theorem on 30 degrees. We can write 30 as 360/12. The theorem says that $\cos (30)=\sqrt{3} / 2$ has degree $\phi(12) / 2=2$ - which is true-and that $\sin (30)=1 / 2$ has degree $\phi(12) / 4=1$ - which is also true. Also, the excluded values 
of $n$ are genuine exclusions. For cosine, we exclude $n=1,2$, corresponding to multiples of 180 degrees. The cosines of such angles are 0 or \pm 1 , hence of degree 1 (and not 1/2). For sine, we exclude $n=4$, corresponding to 90 and 270 degrees, for which sine is 0 , hence of degree 1 (and not $1 / 2$ ).

Note that Lehmer's Theorem implies Gauss's assertion that $\sin \theta$ and $\cos \theta$ are algebraic for every rational angle $\theta$.

Note also the asymmetry between sine and cosine in Lehmer's Theorem. The author finds this unsettling, in light of the complementary angle formula $\sin \theta=$ $\cos \left(90^{\circ}-\theta\right)$.

We apply Lehmer's Theorem to draw up a table. In each row of the following table, we specify a degree $d$ (less than 8). For each degree, we list the denominators $n$ such that $\sin (360 k / n)$ has degree $d$ or $\cos (360 k / n)$ has degree $d$. To construct such a table, one applies Lehmer's Theorem to all the natural numbers $n$ such that sine or cosine of $360 / n$ can have degree at most 7; finding the list of such $n$ 's is an exercise with the definition of $\phi$ given above. (Lehmer's article gives a similar table, but its entries for sine are incorrect because the theorem for sine is not stated correctly there; Niven's book [11] has the correct version of the theorem-reproduced above-but no table. Also, in Lehmer's cosine table there is a 36 that should be a 30.)

TABLE 1: Denominators $n$ such that $\sin 360 k / n$ or $\cos 360 k / n$ have degree $d<8$.

\begin{tabular}{l|ll}
\hline$d$ & sine & cosine \\
\hline 1 & $1,2,4,12$ & $1,2,3,4,6$ \\
2 & $3,6,8,20$ & $5,8,10,12$ \\
3 & 28,36 & $7,9,14,18$ \\
4 & $5,10,16,24,60$ & $15,16,20,24,30$ \\
5 & 44 & 11,22 \\
6 & $7,9,14,18,52,84$ & $13,21,26,28,36,42$ \\
7 & none & none \\
\hline
\end{tabular}

The $d=1$ row of Table 1 says that - for $\theta$ a rational acute angle $-\sin \theta$ is rational if and only if $\theta$ is 30 degrees, and $\cos \theta$ is rational if and only if $\theta$ is 60 degrees. (For a proof of this using trig identities, see [13].) This proves the Governor's Theorem.

The reasoning in the previous paragraph also shows that no rational angle has a sine of $3 / 4$. This answers the question posed in the introduction, albeit in an unsatisfying way.

Now that we understand how to apply Lehmer's Theorem, we prove it. Following the stated goal of this paper, we use Galois theory. A proof that appears more concrete but is really the same can be found in Section 3.4 of [11]. We write $\theta$ for $360 k / n$.

Put

$$
z:=e^{2 \pi i k / n}=\cos \theta+i \sin \theta
$$

a primitive $n$th root of unity in the complex numbers. Then

$$
\cos \theta=\frac{z+\bar{z}}{2},
$$

where $\bar{z}$ denotes the complex conjugate of $z$. Since $k$ and $n$ are relatively prime, there is some natural number $\ell$ such that $k \ell$ is congruent to $n-k(\bmod n)$. Therefore,

$$
\bar{z}=e^{-2 \pi i k / n}=\left(e^{2 \pi i k / n}\right)^{\ell}=z^{\ell} .
$$


In particular, $\bar{z}$ belongs to $\mathbb{Q}(z)$. (Alternatively, we can remember the fact that $\mathbb{Q}(z)$ is a Galois extension of $\mathbb{Q}$.) Now $z$ satisfies the equation

$$
z^{2}-2 z \cos \theta+1=0
$$

because $z \bar{z}=1$, so the dimension of $\mathbb{Q}(z)$ over $\mathbb{Q}(\cos \theta)$ is 1 or 2 . On the other hand, these two field extensions are not the same: $z$ cannot belong to $\mathbb{Q}(\cos \theta)$ because $z$ is not a real number (because $n$ is at least 3$)$ and $\mathbb{Q}(\cos \theta)$ consists of real numbers. That is, the dimension $[\mathbb{Q}(z): \mathbb{Q}(\cos \theta)]$ is 2 . Since the dimension of $\mathbb{Q}(z)$ over $\mathbb{Q}$ is $\phi(n)$ - see e.g. [2, $\$ 13.6$, Cor. 42]-we obtain:

$$
[\mathbb{Q}(\cos \theta): \mathbb{Q}]=\frac{[\mathbb{Q}(z): \mathbb{Q}]}{[\mathbb{Q}(z): \mathbb{Q}(\cos \theta)]}=\frac{\phi(n)}{2} .
$$

This proves the part of Lehmer's Theorem regarding cosine.

The result for sine can be derived from the result for cosine using the complementary angle formula

$$
\sin \left(360 \frac{k}{n}\right)=\cos \left(360 \frac{k}{n}-90\right)=\cos \left(360 \frac{4 k-n}{4 n}\right) .
$$

This part of the proof is identical to the one on p. 38 of Niven's book, so we just sketch it. First, observe that since $n$ is not 4 , the fraction $(4 k-n) /(4 n)$, when put in lowest terms, has denominator at least 3 , so we may indeed apply the cosine result. Next, divide the proof into cases depending on the highest power of 2 - say $2^{e}$ - dividing $n$. For example, if $e=1$, then $(4 k-n) /(4 n)$ in lowest terms has denominator $2 n$, and $\sin (\theta)$ has degree $\phi(2 n) / 2$. But $n=2 m$ for some $m$ odd, so

$$
\frac{\phi(2 n)}{2}=\frac{\phi(4 m)}{2}=\frac{\phi(4) \phi(m)}{2}=\phi(m)=\phi(2) \phi(m)=\phi(n) .
$$

The remaining cases are left to the reader. This completes the proof of Lehmer's Theorem.

For later use, we note that $\mathbb{Q}(z)$ is Galois over $\mathbb{Q}$ with abelian Galois group, as we learned in our Galois theory course. Consequently, every subfield of $\mathbb{Q}(z)$ is also Galois over $\mathbb{Q}$ with abelian Galois group, including $\mathbb{Q}(\cos \theta)$ and any subfield of it.

Remark. The proof of the cosine part of Lehmer's Theorem amounts to the observation that $\mathbb{Q}(\cos \theta)$ is the maximal real subfield of $\mathbb{Q}(z)$, i.e., the intersection of $\mathbb{Q}(z)$ with the real numbers.

\section{An expanded trig table}

Our motivation is to answer the question: Why does the standard trig table include exactly the angles $0,30,45,60$, and 90 degrees? If you want to pick angles to put in a trig table and you know Table 1, it would make sense to include exactly those rational angles $\theta$ such that $\sin \theta$ and $\cos \theta$ both have degree at most $d$ for some choice of $d$. If you do this with $d=2$, you find $n=1,2,3,4,6,8,12$ corresponding to angles that are multiples of $360,180,120,90,60,45$, or 30 degrees. Surprise! You find the standard trig table. This explains why the standard trig table includes exactly the angles that it does.

Taking $d=3$, we find no new angles by Table 1 .

Taking $d=4$, we find new denominators $n=5,10,16,20,24$, corresponding to angles that are multiples of $72,36,22 \frac{1}{2}, 18$, and 15 degrees respectively. An expanded trig table including these angles is given in Table 2 below. 
TABLE 2: An expanded trig table including $72^{\circ}$ and all rational angles $\theta$ between $0^{\circ}$ and $45^{\circ}$ such that $\sin \theta$ and $\cos \theta$ have degree $\leq 4$.

\begin{tabular}{ccc}
\hline$\theta$ (in degrees) & $\sin \theta$ & $\cos \theta$ \\
\hline 0 & 0 & 1 \\
15 & $\frac{-1+\sqrt{3}}{2 \sqrt{2}}$ & $\frac{1+\sqrt{3}}{2 \sqrt{2}}$ \\
18 & $\frac{-1+\sqrt{5}}{4}$ & $\frac{\sqrt{5+\sqrt{5}}}{2 \sqrt{2}}$ \\
$22 \frac{1}{2}$ & $\frac{\sqrt{2-\sqrt{2}}}{2}$ & $\frac{\sqrt{2+\sqrt{2}}}{2}$ \\
30 & $\frac{\sqrt{3} / 2}{2 \sqrt{2}}$ & $\frac{1+\sqrt{5}}{4}$ \\
36 & $\frac{\sqrt{5-\sqrt{5}}}{45}$ & $1 / \sqrt{2}$ \\
72 & $\frac{\sqrt{5+\sqrt{5}}}{2 \sqrt{2}}$ & $\frac{-1+\sqrt{5}}{4}$ \\
\hline
\end{tabular}

This table warrants some remarks. First: How does one compute $\sin \theta$ and $\cos \theta$ for the new angles $\theta$ ? The only difficult one turns out to be $\theta=72^{\circ}$; once $\sin 72^{\circ}$ and $\cos 72^{\circ}$ have been computed, all the other entries can be filled in using the halfangle and complementary-angle formulas and known entries from the standard table. To compute $\sin 72^{\circ}$ and $\cos 72^{\circ}$, we follow the proof of Lehmer's Theorem and put

$$
z:=\cos 72^{\circ}+i \sin 72^{\circ}=e^{2 \pi i / 5} .
$$

Note that $z^{5}=1$, i.e., $z$ is a 5 th root of unity. Since 5 is of the form $2^{2^{1}}+1$, it is a Fermat prime, and Gauss gave a general method for computing $p$ th roots of unity for such primes $p$, see [4, $\S \mathrm{VII}],[3, \S \S 20-27]$, or [17, Ch. 12]. An explicit form of $z$ can be found in almost any book on Galois theory where this method is presented. (Alternatively, one can use trigonometric formulas as in [6, pp. 39, 40] or geometry as in $[\mathbf{1}]$.)

Second, some of the entries are noteworthy. For example, the half-angle formula gives

$$
\sin 15^{\circ}=\sqrt{\frac{1-\cos 30^{\circ}}{2}}=\frac{\sqrt{2-\sqrt{3}}}{2},
$$

but in the table we find $(-1+\sqrt{3}) / 2 \sqrt{2}$. Which is it? Indeed they are equal, since they are positive real numbers whose squares agree. The second version is in the table due to the author's general aversion to "nested" square roots.

We can similarly calculate $\sin 22 \frac{1}{2}^{\circ}$ by applying the half-angle formula to $\cos 45^{\circ}$ and finding 


$$
\sin 22 \frac{1}{2}^{\circ}=\frac{\sqrt{2-\sqrt{2}}}{2} .
$$

This nested expression is the one listed in the table. This is not due to some laziness on the author's part, but rather to mathematical reality: this expression cannot be rewritten to eliminate the nesting. Indeed, in the previous paragraph, the field $\mathbb{Q}((-1+\sqrt{3}) / 2 \sqrt{2})$ is (exercise!) the same as $\mathbb{Q}(\sqrt{2}, \sqrt{3})$, a Galois extension of $\mathbb{Q}$ whose Galois group is the Klein four-group (exercise!). Here the field $\mathbb{Q}(\sqrt{2-\sqrt{2}} / 2)$ is also a Galois extension of $\mathbb{Q}$ of degree 4 , but with Galois group $\mathbb{Z} / 4 \mathbb{Z}$. (This assertion is Exercise 14 in $\S 14.2$ in [2].) The reader who verifies these assertions about the Galois groups will see that they imply that $\sin 22 \frac{1}{2}^{\circ}$ cannot be rewritten so as to remove the nested square root.

Bibliographic references. Hoehn [5] gives a nice geometric derivation of sine and cosine for the angles $15^{\circ}$ and $75^{\circ}$. Generally, one can find $\sin \theta$ and $\cos \theta$ whenever $\theta$ is an integer multiple of 3 degrees once one knows the values for $\theta=36^{\circ}$ and various trig identities, see e.g. [18]. For the general question of unnesting square roots, see [9].

\section{Can we expand the table a little further?}

Continuing the procedure from the previous section, we could consider angles $\theta$ such that $\sin \theta$ and $\cos \theta$ have degree $\leq 5$. By Table 1 , no new angles $\theta$ are found. (In general, for every odd number $d \geq 3$, the angles $\theta$ such that $\sin \theta$ and $\cos \theta$ have degree $\leq d$ are exactly the same angles as those whose sine and cosine have degree $\leq d-1$. Proving this claim is an exercise combining Lehmer's Theorem and the definition of $\phi$ which we leave to the reader.)

If we consider those angles with degree $\leq 6$, there are lots of new angles, but also an ugly phenomenon. Consider the case $n=18$, corresponding to the angle $\theta=20^{\circ}$. Table 1 says that $\cos \theta$ has degree $3,{ }^{2}$ and it is a root of the polynomial $8 x^{3}-6 x-1$, as can be seen by the triple-angle formula. (This fact is familiar from a course on Galois theory, because it is part of the standard proof that the angle $20^{\circ}$ cannot be constructed with straightedge and compass.) The graph of this polynomial is

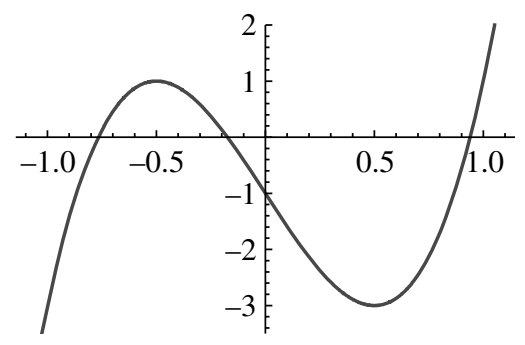

It clearly has three real roots. However, Cardano's formula for finding the roots of a cubic polynomial gives the three seemingly complex roots ${ }^{3}$

$$
x=\sqrt[3]{\frac{1+\sqrt{-3}}{16}}+\sqrt[3]{\frac{1-\sqrt{-3}}{16}} .
$$

\footnotetext{
${ }^{2}$ We only include $20^{\circ}$ at the degree $\leq 6$ stage because $\sin \left(20^{\circ}\right)$ has degree 6 .

${ }^{3}$ Each complex number has three cube roots, so the displayed expression a priori gives 9 complex numbers. The roots of the polynomial $8 x^{3}-6 x-1$ are the values of $x$ where the sum of the two cube roots is a real number.
} 
This is an example of the famous "casus irreducibilis" of the cubic formula, and it is well known that one cannot avoid using complex numbers in writing down these roots (see e.g., $[2, \S 14.7]$ or keep reading this article). Since $\cos \left(20^{\circ}\right)$ is so ugly, we shouldn't include it in our table.

In fact, this example is typical. Write $\theta=360 k / n$ where $k$ and $n$ are relatively prime integers. We have:

THEOREM. The numbers $\cos \theta$ and $\sin \theta$ can be written using only rational numbers, addition, subtraction, multiplication, division, and roots of positive numbers if and only if $\phi(n)$ is a power of 2 .

In the example of 20 degrees above, $\phi(18)$ is 6 , which is not a power of 2 . When we prove the theorem below, we will have in particular proved that cosine of 20 degrees cannot be written using only field operations and roots of positive numbers.

The theorem says that the only candidates for angles $\theta$ to be added to our trig table are those $\theta=360 \mathrm{k} / \mathrm{n}$ where $\phi(n)$ is a power of 2 . Such natural numbers $n$ are precisely those $n$ such that regular $n$-gon is constructible with compass and straightedgeproved by Gauss and Wantzel. Gauss listed the 38 such $n \leq 300$ at the end of [4], and in principle the list is known for $n<2^{2^{22}}+1$ (approximately $2 \times 10^{1262611}$ ), see [12, Seq. A003401].

In the next section, we will investigate what new entries could be added to our trig table. But first we prove the theorem stated above. Write $E$ for the field consisting of real numbers that can be written as in the statement of the theorem, i.e., that can be written using only rational numbers, addition, subtraction, multiplication, division, and roots of positive real numbers.

We first prove the "if" direction, i.e., we suppose that $\phi(n)$ is a power of 2 . This is the easier implication, and the proof proceeds just as for constructibility of a regular $n$-gon. By the observation just after the proof of Lehmer's Theorem, the extension $\mathbb{Q}(\cos \theta)$ of $\mathbb{Q}$ is Galois with abelian Galois group of order $\phi(n) / 2$. Therefore, there is a chain of fields

$$
\mathbb{Q}=K_{0} \subset K_{1} \subset \cdots \subset K_{r}=\mathbb{Q}(\cos \theta)
$$

such that each $K_{i+1}$ is a 2-dimensional extension of $K_{i}$. It follows that $K_{i+1}$ is obtained from $K_{i}$ by adjoining the square root of some element $a_{i} \in K_{i}$. Since $K_{i+1}$ is contained in $\mathbb{Q}(\cos \theta)$ and hence in $\mathbb{R}$, the element $a_{i}$ is positive. We conclude that $\cos \theta$ belongs to $E$. The pythagorean identity $\sin ^{2} \theta+\cos ^{2} \theta=1$ implies that

$$
\sin \theta= \pm \sqrt{1-\cos ^{2} \theta}
$$

hence $\sin \theta$ also belongs to $E$. This completes the proof of the "if" direction.

To prove the more difficult "only if" direction, we suppose that $\phi(n)$ is not a power of 2, i.e., is divisible by an odd prime $p$. Note that this implies that $n$ is at least 7. For sake of contradiction, suppose that $\cos \theta$ or $\sin \theta$ belongs to $E$. In the latter case, the pythagorean identity implies that $\cos \theta$ belongs to $E$, so in any case we have that $\cos \theta$ belongs to $E$. Lehmer's Theorem gives that $p$ divides the dimension of $\mathbb{Q}(\cos \theta)$ over $\mathbb{Q}$. We observed after the proof of that theorem that $\mathbb{Q}(\cos \theta)$ is Galois over $\mathbb{Q}$ with abelian Galois group, so there exists a Galois extension $K$ of $\mathbb{Q}$ contained in $\mathbb{Q}(\cos \theta)$ such that the dimension of $\mathbb{Q}(\cos \theta)$ over $K$ is $p$.

Since $\cos \theta$ belongs to $E$, there is a tower of fields

$$
\mathbb{Q}=F_{0} \subset F_{1} \subset \cdots \subset F_{r} \subset \mathbb{R}
$$

such that $F_{r}$ contains $\cos \theta$ and each $F_{i+1}$ is obtained from $F_{i}$ by adjoining a real $n_{i}$ th root of some positive $a_{i} \in F_{i}$. By inserting additional terms in this tower if necessary, 
we may assume that $n_{i}$ is a prime number for all $i$. Fix $i$ such that the compositum $K F_{i}$ does not contain $\cos \theta$, but $K F_{i+1}$ does.



The field $K F_{i}(\cos \theta)$ properly contains $K F_{i}$ and (since $K$ is Galois over $\mathbb{Q}$ ) the dimension of $K F_{i}(\cos \theta)$ over $K F_{i}$ divides the dimension of $K(\cos \theta)$ over $K$, which is the prime $p$. So $K F_{i}(\cos \theta)$ has dimension $p$ over $K F_{i}$. On the other hand, $K F_{i+1}=K F_{i}\left(\sqrt[n_{i}]{a_{i}}\right)$ is a proper extension of $K F_{i}$ of degree dividing the prime $n_{i}$; since $K F_{i}(\cos \theta)$ is contained in $K F_{i+1}$, we conclude that $n_{i}=p$ and

$$
K F_{i}(\cos \theta)=K F_{i+1}=K F_{i}\left(\sqrt[p]{a_{i}}\right)
$$

is Galois over $K F_{i}$. It follows that $K F_{i+1}$ contains a full set of $p$ th roots of unity. But this is impossible because $p$ is odd and $K F_{i+1}$ consists of real numbers. This contradicts the assumption that $\cos \theta$ or $\sin \theta$ belongs to $E$ and completes the proof of the theorem.

Remarks. (i) One reader of this paper asked what the correct theorem would be if the word "positive" were removed from the statement of the theorem. Or, to restate the question: What rational angles $\theta$ have values of $\cos \theta$ and $\sin \theta$ that are solvable by radicals? In the proof of Lehmer's Theorem, we saw that $\cos \theta$ generates an abelian extension of $\mathbb{Q}$, so the answer is "all rational angles $\theta$ " by Galois's criterion.

(ii) The proof of the difficult direction of the theorem did not involve the result about constructibility of regular $n$-gons, despite the similarity in the two statements. Indeed, the restriction that $\phi(n)$ be a power of 2 arose here because $\phi(n)$ is-up to multiplication by a power of 2 -the degree of a real Galois extension $Q(\cos \theta)$ of $Q$ obtained by taking roots of positive real numbers. This implied that $Q(\cos \theta)$ can be obtained by adjoining only square roots, hence that $\phi(n)$ is a power of 2 . In contrast, in the theorem about $n$-gons, you begin with the hypothesis that you can only take square roots.

(iii) A more general version of the proof above is given in [7] or with Exercises $12-14$ in $\S 14.7$ of [2]. Our proof is much simpler because the number that we assume belongs to $E$-namely, $\cos \theta$ - generates a Galois extension of $\mathbb{Q}$.

\section{What the next few numbers look like}

By the previous section, our trig table should only include angles $360 k / n$ where $\phi(n)$ is a power of 2. If we want to expand Table 2, how should we do it? 
We have already considered the denominators $n=1,2, \ldots, 6,8,10,12,16,20,24$. The smallest natural number $n$ not in that list and with $\phi(n)$ a power of 2 is $n=15$, corresponding to angles that are multiples of 24 degrees. To compute $\sin 24^{\circ}$ and $\cos 24^{\circ}$, we can use the familiar trick from the construction of regular $n$-gons. Namely, factor 15 as $3 \cdot 5$ and recall that we know $e^{2 \pi i / 3}$ and $e^{2 \pi i / 5}$ because we know the values of sine and cosine for $120^{\circ}$ and $72^{\circ}$, so we can explicitly compute

$$
e^{2 \pi i / 3} \cdot e^{2 \pi i / 5}=e^{2 \pi i \cdot 8 / 15} \text {. }
$$

Moreover, $2 \cdot 8=15+1$, so

$$
\left(e^{2 \pi i \cdot 8 / 15}\right)^{2}=e^{2 \pi i / 15} .
$$

Performing these computations and extracting the real part, we find:

$$
8 \cos 24^{\circ}=(1+\sqrt{5})+(\sqrt{5}-1) \sqrt{\frac{3}{2}(5+\sqrt{5})}=(1+\sqrt{5})+2 \sqrt{\frac{3}{2}(5-\sqrt{5})} .
$$

The next $n$ for which the corresponding $\theta$ does not appear in Table 2 is $n=17$, corresponding to the angle $\theta=21 \frac{3}{17}^{\circ}$. Seventeen is a Fermat prime, and the values of sine and cosine can be computed by Gauss's algorithm. Gauss himself gave the following explicit formula for $\cos \theta$ in Article 365 of [4]:

$$
\begin{aligned}
16 \cos 21 \frac{3}{17}^{\circ}= & -1+\sqrt{17}+\sqrt{34-2 \sqrt{17}} \\
& +2 \sqrt{17+3 \sqrt{17}-\sqrt{34-2 \sqrt{17}}-2 \sqrt{34+2 \sqrt{17}}}
\end{aligned}
$$

These numbers look pretty ugly! Another strategy is to apply a friendly trig identity like a half-angle formula to entries in the table. Doing this with 18 degrees, we find for example that

$$
\cos 9^{\circ}=\frac{1}{2} \sqrt{2+\sqrt{\frac{5+\sqrt{5}}{2}}}
$$

The reader is encouraged to continue along these lines until their personal limits of expression complexity are attained.

\section{Summary}

We observed that we can construct a trig table for each natural number $d$ by including precisely those rational angles $\theta$ such that $\cos \theta$ and $\sin \theta$ are algebraic of degree at most $d$. For $d=1$, the table only included multiples of $90^{\circ}$. For $d=2$, we got the standard trig table consisting of multiples of $30^{\circ}$ and $45^{\circ}$. For $d=4$, we found a larger table, and we exhibited a portion of it in Table 2 . Unfortunately, for $d \geq 6$, it is impossible to write the cosine of some angles without using complex numbers. Further investigation revealed that, without using complex numbers, we can only write down $\cos \theta$ and $\sin \theta$ if $\phi(n)$ is a power of 2 , where $n$ is the denominator of $\theta$. The proof of this last fact was different from the similar-sounding result about constructibility of regular $n$-gons with straightedge and compass. 


\section{REFERENCES}

1. B. Bradie, Exact values for the sine and cosine of multiples of $18^{\circ}$ - a geometric approach, College Math. J. 33 (2002) 318-319.

2. D. S. Dummit and R. M. Foote, Abstract Algebra, 3rd ed., Wiley, 2004.

3. H. M. Edwards, Galois Theory, Springer, 1984.

4. C. F. Gauss, Disquisitiones Arithmeticae, trans. by A. A. Clarke, rev. by W. C. Waterhouse, Springer, 1986.

5. L. Hoehn, Proof without words, College Math. J. 35 (2004) 282.

6. H. E. Huntley, The Divine Proportion: A Study in Mathematical Beauty, Dover, 1970.

7. I. M. Isaacs, Solution of polynomials by real radicals, Amer. Math. Monthly 92 (1985) 571-575.

8. N. Jacobson, Basic Algebra 1, 2nd ed., W. H. Freeman, 1985.

9. S. Landau, How to tangle with a nested radical, Math. Intelligencer 16(2) (1994) 49-55.

10. D. H. Lehmer, A note on trigonometric algebraic numbers, Amer. Math. Monthly 40 (1933) 165-166.

11. I. Niven, Irrational Numbers, Carus Mathematical Monographs, vol. 11, Mathematical Association of America, 1956.

12. N. Sloane et al., Online encyclopedia of integer sequences, online at http://www.research.att.com/ njas/sequences/Seis.html

13. J. M. H. Olmsted, Rational values of trigonometric functions, Amer. Math. Monthly 52 (1945) 507-508.

14. M. Schneider, Teenager stumps Bush with pop math quiz, Associated Press, 6 July 2004.

15. D. O. Shklarsky, N. N. Chentzov, and I. M. Yaglom, The USSR Olympiad Problem Book: Selected Problems and Theorems of Elementary Mathematics, Dover, 1994.

16. St. Petersburg Times (FL), Query stumps governor, but it wasn't easy, was it?, 7 July 2004.

17. J.-P. Tignol, Galois' Theory of Algebraic Equations, World Scientific, 2001.

18. Wikipedia, Exact trigonometric constants, available at http://en.wikipedia.org/wiki, version 12 November 2006. 